\title{
The Wisconsin H $\alpha$ Mapper (WHAM): A Brief Review of Performance Characteristics and Early Scientific Results
}

\author{
R. J. Reynolds ${ }^{1}$, S. L. Tufte ${ }^{1}$, L. M. Haffner ${ }^{1}$, \\ K. Jaehnig ${ }^{2}$ and J. W. Percival ${ }^{2}$ \\ ${ }^{1}$ Department of Astronomy, University of Wisconsin, \\ 475 N. Charter St., Madison, WI 53706, USA \\ ${ }^{2}$ Space Astronomy Laboratory, University of Wisconsin, \\ 1150 University Ave, Madison, WI 53706, USA \\ Received 1997 August 15, accepted 1997 December 2
}

\begin{abstract}
The Wisconsin H $\alpha$ Mapper (WHAM) is a recently completed facility for the detection and study of faint optical emission lines from diffuse ionised gas in the disk and halo of the Galaxy. WHAM consists of a $15 \mathrm{~cm}$ diameter Fabry-Perot spectrometer coupled to a $0.6 \mathrm{~m}$ 'telescope', which provide a $1^{\circ}$ diameter beam on the sky and produce a $12 \mathrm{~km} \mathrm{~s}^{-1}$ resolution spectrum within a $200 \mathrm{~km} \mathrm{~s}^{-1}$ spectral window. This facility is now located at Kitt Peak in Arizona and operated remotely from Madison, Wisconsin, $2400 \mathrm{~km}$ distant. Early results include a velocity-resolved $\mathrm{H} \alpha$ map of a $70^{\circ} \times 100^{\circ}$ region of the sky near the Galactic anticentre, the first detections of $\mathrm{H} \alpha$ emission from the M I and A high velocity clouds, and the first detections of [O I] $\lambda 6300$ and other faint 'diagnostic' lines from the warm ionised medium. Through the summer of 1998, WHAM will be devoted almost exclusively to a survey of the northern sky, which will provide maps of the distribution and kinematics of the diffuse HII through the optical $\mathrm{H} \alpha$ line in a manner that is analogous to earlier sky surveys of the HI made through the $21 \mathrm{~cm}$ line.
\end{abstract}

Keywords: ISM: general — ISM: HII regions — ISM: structure — instrumentation: spectrographs

\section{Introduction}

Warm $\left(10^{4} \mathrm{~K}\right)$, ionised hydrogen is a principal component of our Galaxy's interstellar medium, with a mass surface density approximately one-third that of the HI, a vertical scale height nearly ten times that of the HI cloud layer, and a power budget equal to the kinetic energy injected into the interstellar medium by supernovae (Kulkarni \& Heiles 1987; Reynolds 1991). Detailed studies of this component are thus required for an accurate understanding of interstellar matter and processes, as well as for a proper interpretation of other astrophysical observations affected by this foreground screen of HII. Although originally detected in the 1960 s with radio techniques (e.g. Hoyle \& Ellis 1963; Bridle \& Venugopal 1969), subsequent developments in Fabry-Perot spectroscopy soon showed that the primary source of information about the distribution, kinematics, and other properties of this gas is through the detection and study of interstellar emission lines at optical wavelengths (e.g. Reynolds, Scherb \& Roesler 1973; Reynolds 1980). However, the emission lines are weak, and a comprehensive exploration of this component had to await the development of modern, low-noise imaging detectors, which increased dramatically the efficiency of spectrometers and imaging cameras for observations of the faint interstellar emission.

\section{The Wisconsin $\mathrm{H} \alpha$ Mapper}

We have recently built a Fabry-Perot spectrometer called the Wisconsin $\mathrm{H} \alpha$ Mapper (WHAM), which uses a low-noise, high-efficiency CCD camera as a multichannel detector behind a pair of $15 \mathrm{~cm}$ diameter Fabry-Perots. The well known advantage of the Fabry-Perot spectrometer is that at a specified spectral resolving power it has a throughput $\sim 100$ times larger than that of a grating spectrometer with a dispersing element of the same area. This angular throughput advantage over the grating spectrometer makes the Fabry-Perot well suited for high spectral resolution studies of faint extended emission-line sources, particularly when coupled to an imaging detector such as a CCD, which can record many spectral elements simultaneously. In this case, when collimated rays passing through the Fabry-Perot are imaged on the detector, a 'ring spectrum' is formed in an azimuthally symmetric pattern about 


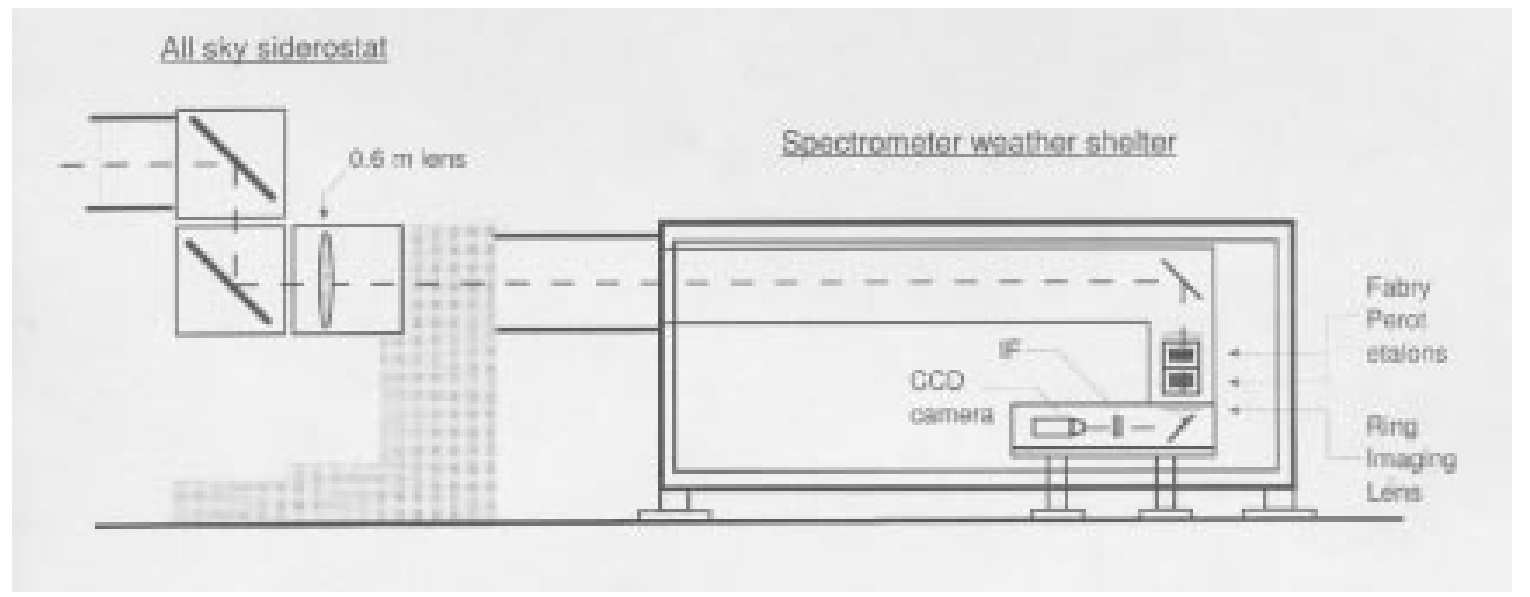

Figure 1-Schematic diagram of WHAM, showing the siderostat and the trailer that shelters the spectrometer. In this diagram the siderostat is pointing at the southern horizon.

the optical axis, with wavelength decreasing from the centre to the edge such that equal spectral intervals correspond to a set of equal-area, nested annuli on the detector (e.g. Reynolds et al. 1990; Tufte 1997).

The spectral and spatial properties of the diffuse interstellar emission-line background, determined from earlier observations, have defined the specific characteristics of the WHAM system. Because the interstellar emission is weak, the throughput was maximised by incorporating Fabry-Perot etalons with the largest available clear aperture $(14 \cdot 7 \mathrm{~cm})$. Furthermore, the detector is a high quantum efficiency $(78 \%$ at $\mathrm{H} \alpha)$, low-noise $\left(3 \mathrm{e}^{-} \mathrm{rms}\right)$ CCD camera. WHAM is a dual-etalon spectrometer, which greatly reduces multi-order ghosts, especially those arising from the relatively strong atmospheric $\mathrm{OH}$ emission lines within the passband of the interference filter. The tandem etalon design also suppresses the Lorentzian-like wings that are typical of single etalon systems, making it easier to detect the relatively weak and broad interstellar features in the wing of the ever-present geocoronal $\mathrm{H} \alpha$ line. The spectral resolution of $12 \mathrm{~km} \mathrm{~s}^{-1}$ matches well the $25 \mathrm{~km} \mathrm{~s}^{-1}$ widths (FWHM) of the narrowest interstellar $\mathrm{H} \alpha$ emission components, and the $200 \mathrm{~km} \mathrm{~s}^{-1}$ spectral window is set by the radial velocity spread of the emission components, which are typically within $\pm 50-70 \mathrm{~km} \mathrm{~s}^{-1}$ of the local standard of rest (LSR). Broadband, antireflection $(<0 \cdot 5 \%)$ coatings on most of the optical surfaces suppress extraneous reflections, which together with multilayer broadband reflection ( $\simeq 90 \%)$ coatings on the etalons permit observations anywhere within the spectral region 4800 to $7200 \AA$. WHAM's $4.4 \AA\left(200 \mathrm{~km} \mathrm{~s}^{-1}\right)$ spectral window can be centered on any wavelength within this region by using a gas $\left(\mathrm{SF}_{6}\right)$ pressure control system to tune each etalon and a filter wheel to provide the correct isolating interference filter. The angular resolution of $1^{\circ}$ is adequate to resolve some of the spatial structure known to exist in the $\mathrm{H} \alpha$ background at high Galactic latitudes (e.g. Reynolds 1993) and to create $\mathrm{H} \alpha$ maps comparable to existing $21 \mathrm{~cm}$ surveys. (a)

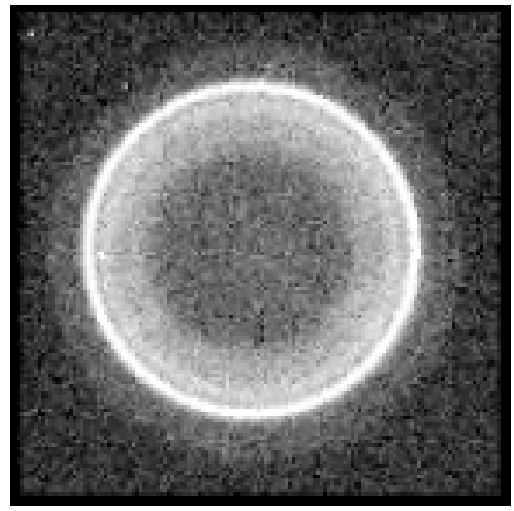

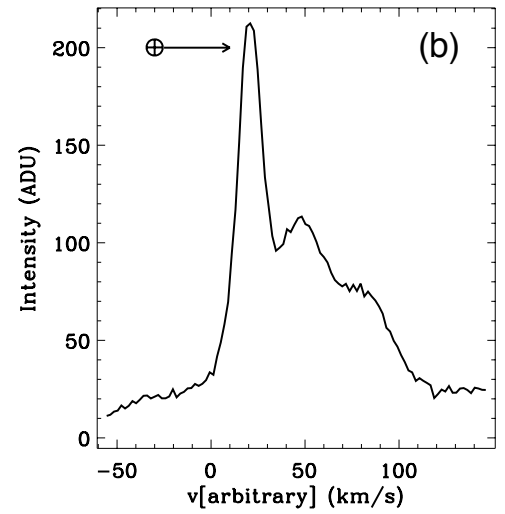

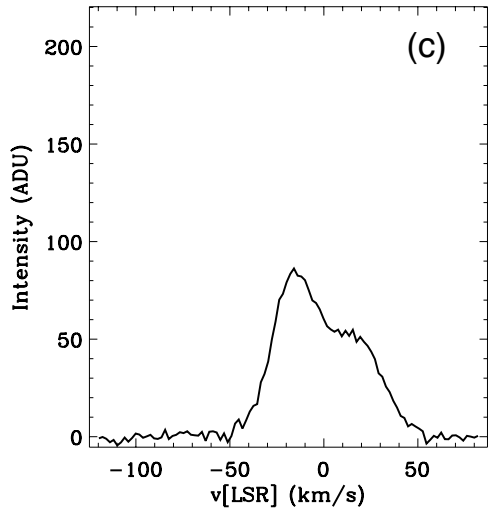

Figure 2-WHAM $\mathrm{H} \alpha$ data for $l=203 . .^{\circ} 8, b=-43 .^{\circ} 3$. (a) the raw CCD camera image (30 s exposure); (b) the spectrum produced by annular binning of the CCD image ( $\oplus$ denotes the geocoronal line); (c) the final, pure interstellar $\mathrm{H} \alpha$ spectrum after flat fielding, the removal of the geocoronal line, and the subtraction of the sky background continuum. The intensities of the two blended interstellar lines near $-15 \mathrm{~km} \mathrm{~s}^{-1}$ and $+15 \mathrm{~km} \mathrm{~s}^{-1}$ (LSR) are approximately $4 \mathrm{R}$ and $2 \mathrm{R}$, respectively. 
A simplified diagram of the WHAM optical system is shown in Figure 1. Light from the sky is directed by the flat mirrors of a two-axis siderostat horizontally through a $0.6 \mathrm{~m}$ diameter, $8.6 \mathrm{~m}$ focal length objective lens into a $2.5 \mathrm{~m} \times 2.5 \mathrm{~m} \times 6$ $\mathrm{m}$ trailer that contains the spectrometer. The sky is imaged between the two Fabry-Perot etalons, whose $15 \mathrm{~cm}$ diameters define WHAM's $1^{\circ}$ diameter beam on the sky. The light then passes through a series of spectral imaging lenses, a broadband $(20 \AA)$ interference filter, and a high-speed camera lens, which images the 'ring spectrum' onto the CCD chip. In this normal spectral mode the sky is not imaged on the detector, only the average spectrum within the beam. This eliminates any possible confusion between spectral features and the spatial structure of the source, including stars, within the beam. With the insertion of additional lenses (not shown) into the optical path, the WHAM spectrometer can create, instead of a spectrum, a narrowband, monochromatic image of the sky at about $1^{\prime}$ angular resolution within the $1^{\circ}$ beam. The spectral width of the sky image can be adjusted by an iris diaphragm to any value between $10 \mathrm{~km}$ $\mathrm{s}^{-1}(0 \cdot 2 \AA$ at $\mathrm{H} \alpha)$ and $200 \mathrm{~km} \mathrm{~s}^{-1}(4 \cdot 4 \AA)$. The narrowest setting allows the imaging of individual velocity components within an interstellar emission profile, or with a series of exposures at different radial velocities, the creation of a complete data cube for the $1^{\circ}$ field.

WHAM has been located at Kitt Peak, Arizona, since November 1996. The siderostat, the CCD camera and $\mathrm{LN}_{2}$ dewar filling system, the etalon pressure-tuning system, the interference filter wheel, the calibration light sources, the imaging optics carriage and iris, plus a number of environmental sensors provide information to and can be commanded from a single workstation. By incorporating and extending the remote observing subsystems developed for the 3.5 m WIYN telescope (Percival 1994), the entire WHAM facility, including opening and closing at the beginning and end of the observing night, is operated from a campus office at the University of Wisconsin in Madison, $2400 \mathrm{~km}$ from Kitt Peak.

\section{Early Results}

\section{$3.1 \mathrm{H \alpha}$ Sky Survey}

Between January 1997 and September 1998, WHAM will carry out one of its primary missions, a northern sky $\mathrm{H} \alpha$ survey of the warm ionised medium. This survey will consist of approximately 33,000 spectra above declination $-20^{\circ}$, sampling the sky on a $0^{\circ} .85 \times 0^{\circ} .98$ grid with a $1^{\circ}$ beam. Each spectrum has a $30 \mathrm{~s}$ integration time and covers a $4.4 \AA$ $\left(200 \mathrm{~km} \mathrm{~s}^{-1}\right)$ spectral interval centred near the LSR at a resolution of $0 \cdot 26 \AA\left(12 \mathrm{~km} \mathrm{~s}^{-1}\right)$. All observations are carried out during dark of the moon to avoid contamination by features in the solar spectrum. This survey will provide for the first time a detailed view of the distribution and kinematics of the diffuse ionised hydrogen through the optical $\mathrm{H} \alpha$ line, comparable to the large-scale survey maps of the neutral hydrogen obtained through the radio $21 \mathrm{~cm}$ line.

Figure 2 illustrates a sample spectrum from the survey at $l=203^{\circ} .8, b=-43^{\circ} .3$, showing both the raw $\mathrm{CCD}$ image and the resulting $\mathrm{H} \alpha$ spectra. The geocoronal line is the thin, bright annulus in the CCD 'ring spectrum', which appears as a prominent, relatively narrow emission line in the centre frame of Figure 2. The interstellar emission is the broader feature inside the geocoronal ring, appearing in this case to consist of two blended velocity components at $+30 \mathrm{~km} \mathrm{~s}^{-1}$ and $+60 \mathrm{~km} \mathrm{~s}^{-1}$ with respect to the geocoronal line. In general, the separation between the interstellar emission and the geocoronal line is due to a combination of the earth's orbital velocity, the sun's peculiar velocity, and intrinsic motions of the interstellar gas, including Galactic differential rotation. The two interstellar components have intensities of about $2 \mathrm{R}$ and $4 \mathrm{R}$, where a Rayleigh is $1 \mathrm{R}=2 \cdot 41 \times 10^{-7} \mathrm{erg} \mathrm{cm}{ }^{-2} \mathrm{~s}^{-1} \mathrm{sr}^{-1}$ at $\mathrm{H} \alpha$, and corresponds to an emission measure of $2 \cdot 3 \mathrm{~cm}^{-6} \mathrm{pc}$ for a temperature of $8000 \mathrm{~K}$ (Reynolds 1991). The geocoronal line is removed from the data by fitting each spectrum with gaussian components and then subtracting from the spectrum the fitted gaussian associated with the geocorona. The resulting pure interstellar spectrum is shown in the third frame of Figure 2. The absolute intensity calibration is obtained by comparison with standard astronomical sources (e.g. Scherb 1981).

A portion of the survey data is presented in Figure 3 as a grey-scale map of the total intensity of the interstellar $\mathrm{H} \alpha$ emission. This map, synthesised from approximately $7000 \mathrm{H} \alpha$ spectra, covers the region of the sky between about $170^{\circ}$ to $240^{\circ}$ Galactic longitude and $\pm 50^{\circ}$ Galactic latitude. The greyscaling and stretch have been adjusted to reveal the fainter high-latitude emission along with the brighter regions near the plane. Interstellar $\mathrm{H} \alpha$ emission is detected in every direction, with intensities that range from thousands of Rayleighs near the Orion nebula $\left(\sim 209^{\circ},-20^{\circ}\right)$ and $\sim 100-200 \mathrm{R}$ in Barnard's loop and the large $\lambda$ Ori HII region $\left(195^{\circ},-13^{\circ}\right)$, to $\sim 0.5 \mathrm{R}$ in some of the fainter high-latitude regions (e.g. $\left.220^{\circ},+45^{\circ}\right)$. The map reveals numerous large-scale filaments superposed on a fainter $\mathrm{H} \alpha$ background. A number of 'classical' HII regions also dot the map near the Galactic equator. Some of the filamentary features are associated with the OrionEridanus bubble (Reynolds \& Ogden 1979; Sivan 1974), which fills the sky from $l=180^{\circ}$ to $210^{\circ}$ and $b=-10^{\circ}$ to $-50^{\circ}$ and includes Barnard's Loop near its northern boundary. Many of the Orion-Eridanus 
filaments appear to be correlated with emission features at $21 \mathrm{~cm}$ and X-ray wavelengths (see, for example, Brown, Hartmann \& Burton 1995; Burrows et al. 1993; Reynolds \& Ogden 1979). Other filaments on the map have no obvious correspondence to any previously known structures, for example, the $\sim 10 \mathrm{R}$ feature that extends north from $l=220^{\circ}, b=-30^{\circ}$ across the Galactic midplane to $215^{\circ},+5^{\circ}$ or the fainter ( $1 \mathrm{R})$ feature rising vertically from $226^{\circ}$, $+10^{\circ}$ to $229^{\circ},+50^{\circ}$. Narrow velocity interval maps have also been constructed from these data, revealing significant kinematic variations among the various emission features (Haffner et al. 1998, in preparation).

\subsection{High Velocity Clouds}

Although they have been observed via their $21 \mathrm{~cm}$ emission for many years, the origin of the high velocity clouds (HVCs) is still not known (Wakker et al. 1996). The detection of HVCs in $\mathrm{H} \alpha$ (Kutyrev \& Reynolds 1989; Songaila, Bryant \& Cowie 1989; Tufte et al. 1996) and [S II] $\lambda 6716$ (Tufte 1997) has opened a new window through which to explore the nature of these objects. Furthermore, the M I and A clouds have been found to be located far above the Galactic midplane, $z=1 \cdot 5-4 \cdot 4 \mathrm{kpc}$ (Danly, Albert \& Kuntz 1993) and 3-7 kpc (Wakker et al. 1996; van Woerden et al., personal communication), respectively, making $\mathrm{HVCs}$ excellent probes of the environment outside the Galactic disk. WHAM has detected $\mathrm{H} \alpha$ from the M I, M II, A, and C clouds with an intensity of $\sim 0 \cdot 1 \mathrm{R}$ (Tufte et al. in preparation). If the HII in these clouds is produced by photoionisation, then the required ionising flux is $1-2 \times 10^{5}$ photons $\mathrm{cm}^{-2} \mathrm{~s}^{-1}$ (Tufte 1997), which is about $5-10 \%$ of that required to produce the warm ionised component of the interstellar medium in the disk. Observations in many directions toward and near the M I cloud suggest that the HII is confined primarily to the region of $21 \mathrm{~cm}$ emission (at least in projection) and does not form an extended halo about the cloud (Tufte et al. 1996).

\subsection{Diagnostic Lines}

In addition to $\mathrm{H} \alpha$, WHAM is able to observe other emission lines that probe the temperature

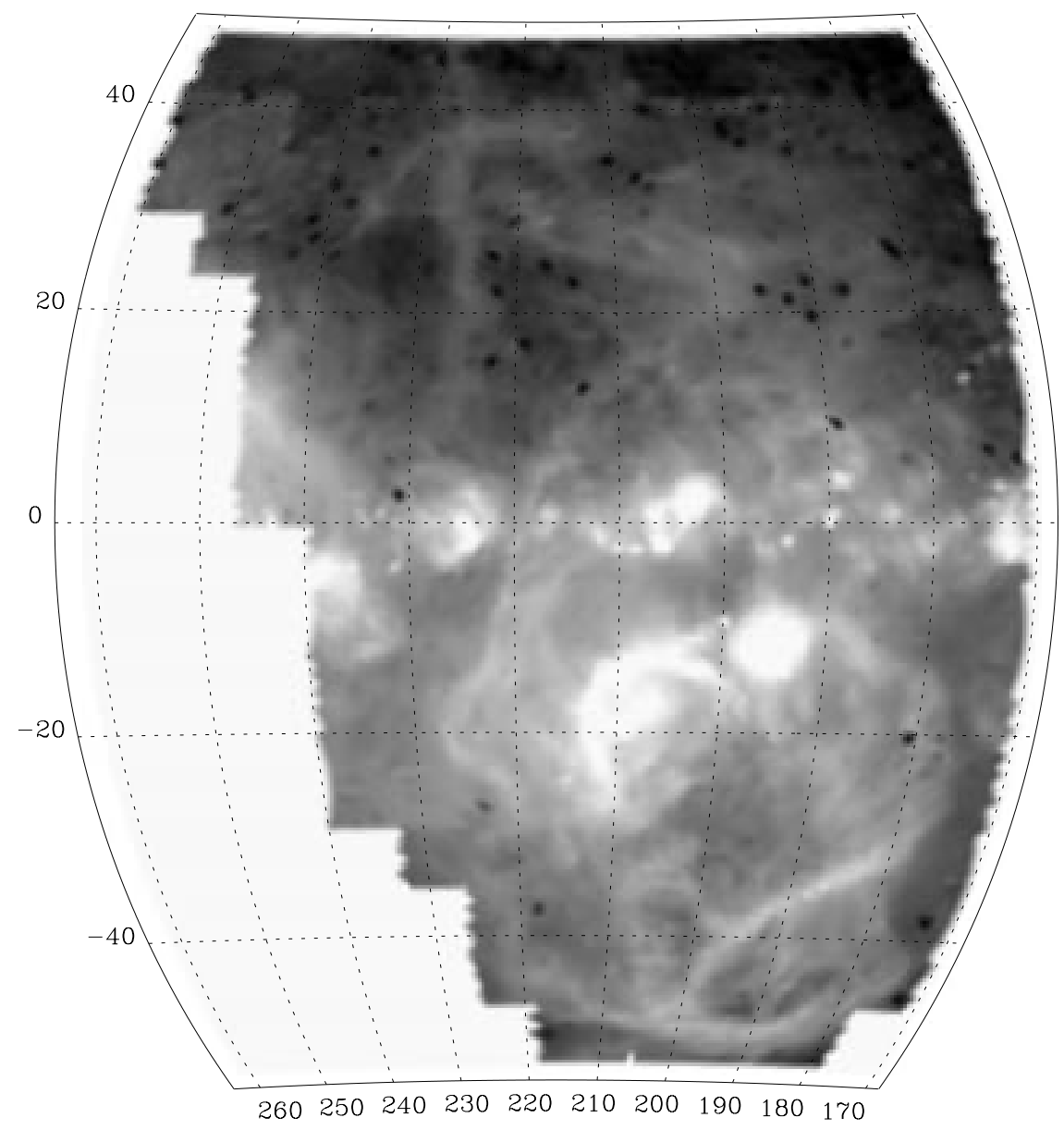

Figure 3-Total intensity map of the diffuse interstellar $\mathrm{H} \alpha$ background in the Galactic anticenter region (extracted from the WHAM sky survey). The map is in Galactic coordinates, with the Galactic equator running horizontally through the center. The map is bounded on the left by the $-20^{\circ}$ declination limit of the survey. Barnard's Loop is the bright arc near $210^{\circ},-15^{\circ}$ and the large $\lambda$ Ori HII region is centered near $195^{\circ},-12^{\circ}$. The small black dots are pixels with missing data or spectra contaminated by a bright star within the beam. 
and ionisation conditions within the gas, including [S II] $\lambda 6716,[\mathrm{~N} \mathrm{II]} \lambda 6584,[\mathrm{~N} \mathrm{II}] \lambda 5755, \mathrm{He} \mathrm{I} \lambda 5876$, [O I] $\lambda 6300$, and [O III] $\lambda 5007$ (Tufte et al. 1996; Haffner, Reynolds \& Tufte 1996; Reynolds et al. 1997). Some of these lines are too faint to have been detected previously. For example, the $[\mathrm{O} \mathrm{I}] \lambda 6300$ line intensity relative to $\mathrm{H} \alpha$ is a measure of the amount of neutral hydrogen within the warm ionised regions, and provides an important constraint on photoionisation models (e.g. Domgörgen \& Mathis 1994; Reynolds 1989). Observations with WHAM have provided the first detections of this line from the diffuse interstellar medium, with an intensity that varies from less than $1 \%$ to about $4 \%$ of that of the interstellar H $\alpha$ (Reynolds et al. 1998). According to the photoionisation models (Domgörgen \& Mathis 1994), these low $[\mathrm{O} \mathrm{I}] / \mathrm{H} \alpha$ intensity ratios suggest that most of the $\mathrm{H} \alpha$ originates from density-bounded, nearly fully ionised regions rather than from partially ionised HI clouds or HII 'skins' on the surfaces of HI clouds.

\section{Summary and Conclusions}

The $1^{\circ}$ resolution WHAM $\mathrm{H} \alpha$ survey plus follow-up observations of fainter diagnostic lines will provide new information about the composition, structure, and kinematics of the warm ionised component of the interstellar medium and the sources of ionisation within the Galactic disk and halo. In addition, it will provide the opportunity to compare the distribution and kinematics of the warm ionised gas with other major components of the interstellar medium, particularly the HI, as well as other diffuse emission, such as the microwave background. Finally, because WHAM can spectrally resolve the Galactic emission from contaminating terrestrial emission lines, the WHAM survey will be a source of kinematic and absolute intensity information for complementary, higher angular resolution $\mathrm{H} \alpha$ studies carried out at lower spectral resolution.

\section{Acknowledgments}

This work is supported primarily through grants from the National Science Foundation (AST91-15703; AST91-22701; AST96-19424), with additional support from the Graduate School, Department of Astronomy, and Department of Physics at the University of Wisconsin-Madison. The WHAM spectrometer and siderostat were built at the University of Wisconsin Space Astronomy Laboratory and the Physical Sciences Laboratory, respectively. We are also grateful to Fred Roesler, John Harlander, Frank Scherb, Alexander Kutyrev, and Nicole Hausen, who provided helpful advice and valuable assistance at various times during the design, construction, and testing phases of the WHAM facility.

Bridle, A. H., \& Venugopal, V. P. 1969, Nature, 224, 545 Brown, A. G. A., Hartmann, D., \& Burton, W. B. 1995, A\&A, 300, 903

Burrows, D. N., Singh, K. P., Nousek, J. A., Garmire, G. P., \& Good, J. 1993, ApJ, 406, 97

Danly, L., Albert, C. E., \& Kuntz, K. D. 1993, ApJ, 416, L29

Domgörgen, H., \& Mathis, J. S. 1994, ApJ, 428, 647

Haffner, L. M., Reynolds, R. J., \& Tufte, S. L. 1996, Bull. AAS, 28, 890

Haffner, L. M., Reynolds, R. J., \& Tufte, S. L. 1998, Faint large-scale $\mathrm{H} \alpha$ filaments in the Milky Way, in preparation

Hoyle, F., \& Ellis, G. R. A. 1963, Aust. J. Phys., 16, 1

Kulkarni, S. R., \& Heiles, C. 1987, in Interstellar Processes, ed. D. J. Hollenbach \& H. A. Thronson Jr (Dordrecht: Reidel), p. 87

Kutyrev, A. S., \& Reynolds, R. J. 1989, ApJ, 344, L11

Percival, J. W. 1994, Bull. AAS, 26, 1422

Reynolds, R. J. 1980, ApJ, 236, 153

Reynolds, R. J. 1989, ApJ 345, 811

Reynolds, R. J. 1991, in IAU Symp. 144, The Interstellar Disk-Halo Connection in Galaxies, ed. H. Bloemen (Dordrecht: Kluwer), p. 67

Reynolds, R. J. 1993, in Back to the Galaxy, AIP Conf. Proc. No. 278, ed. S. S. Holt \& F. Verter (New York: AIP), p. 156

Reynolds, R. J., Hausen, N. R., Tufte, S. L., \& Haffner, L. M. 1998, ApJ, 494, L99

Reynolds, R. J., \& Ogden, P. M. 1979, ApJ, 229, 942

Reynolds, R. J., Roesler, F. L., Scherb, F., \& Harlander, J. 1990, in Instrumentation in Astronomy, SPIE Proc. Vol. 1235, ed. D. Crawford (Billingham, WA: SPIE), p. 610

Reynolds, R. J., Scherb, F., \& Roesler, F. L. 1973, ApJ, 185,869

Scherb, F. 1981, ApJ, 243, 644

Sivan, J.-P. 1974, A\&AS, 16, 173

Songaila, A. A., Bryant, W., \& Cowie, L. L. 1989, ApJ, 345, L71.

Tufte, S. L. 1997, PhD thesis, University of WisconsinMadison

Tufte, S. L., Reynolds, R. J., Haffner, L. M., \& Jaehnig, K. P. 1996, Bull. AAS, 28, 890

Wakker, B., et al. 1996, ApJ, 473, 834 